\title{
IN MEMORIAM: ANNE SJERP TROELSTRA 1939-2019
}

Anne Troelstra was born on August 10, 1939 in Maartensdijk (Utrecht) and grew up in Eindhoven, where he attended the Lorentz Lyceum. In 1957 he enrolled as a mathematics student at the University of Amsterdam, the university where he was to spend most of his working life, and where he was still seen regularly right up to his last illness. Fellow students with whom he was close included Olga Bakker and E.W. Beth's students Dick de Jongh and Hans Kamp. In high school Anne had already read "Intuitionism: An Introduction," Arend Heyting's engaging exposition of Brouwer's mathematical philosophy. In 1964 he finished his master's studies cum laude and was immediately appointed assistant professor, as was then the custom with excellent students. In 1965 Anne and Olga were married, and in 1966 Anne completed his Ph.D. dissertation on intuitionistic topology under Heyting's supervision.

The next two years were of great importance for Anne's mathematical development and professional career. Interested in the metamathematics of choice sequences, he traveled with Olga to Stanford University for the academic year 1966-67, on a stipend from the Netherlands Organization for the Advancement of Science (then called ZWO). At Stanford he followed lectures by Solomon Feferman and Dana Scott, with Jon Barwise and Kenneth Kunen among their students, altogether a very stimulating environment. His main influence there, however, was Georg Kreisel whose concept of "informal rigour" (rigorous concept analysis) remained a guiding principle for Anne in his later investigations.

Kleene's and Vesley's "Foundations of Intuitionistic Mathematics, especially in relation to recursive functions" (1965) developed a formal system I for analysis in close accordance with Brouwer's controversial ideas about choice sequences, in particular with his claim that all total functions on Baire space are continuous. Kleene used realizability to prove the consistency of I relative to a neutral, classically correct subsystem B.

Kreisel proposed a new sort of sequence he called "lawless" in contrast to Brouwer's "lawlike" sequences, intending to use both to analyze the notion of choice sequence. Anne corrected Kreisel's axioms for lawless sequences (LS) and choice sequences determined by a spread (CS), and proved finitistically that CS is a conservative extension of its neutral lawlike subsystem IDB. In Amsterdam, in 1967, he was an invited speaker, alongside Kleene and Kreisel, at the Congress for Logic, Methodology and Philosophy of Science. His paper "The theory of choice sequences" in the conference proceedings announced the conservativity result; its proof appeared in 1970 in a joint paper with Kreisel.

In the summer of 1968 Anne gave a fundamental course of ten lectures at the conference on Intuitionism and Proof Theory at SUNY Buffalo, where most logicians who were then interested in these fields were gathered. The content of these lectures was quickly integrated into his first book: "Principles of Intuitionism."

In 1968, the year Olga's and Anne's first daughter Willemien was born, Anne was appointed Lector (associate professor) in analysis at the University of Amsterdam. In 1970, when their second daughter Ine was born, he was offered the chair of mathematics which had been held by Heyting, as successor to the great Dutch mathematician-philosopher L. E. J. Brouwer. This position, which Anne held until his retirement in 2000, brought with it the responsibility to inspire interest and progress in intuitionistic logic and mathematics, a challenge he took very seriously. Although he retained a lifelong interest in Brouwer's concept of choice sequence, by his investigations and his influence on students and colleagues Anne extended the Dutch tradition into new directions, giving intuitionism a central place in metamathematical research. 
While lecturing, mentoring graduate students and publishing papers, he helped to organize conferences in the Netherlands and at Oberwolfach. Among his books are the essential "Metamathematical Investigations of Intuitionistic Arithmetic and Analysis" (Springer Lecture Notes 344, 1973) with important supplementary chapters by Smorynski, Zucker and Howard, and "Constructivism in Mathematics" I and II (with D. van Dalen, 1988). In 1976 Anne was elected a member of the Dutch Academy of Sciences. In 1996 he was awarded the F. L. Bauer prize of the Technical University of München for internationally outstanding contributions to theoretical computer science, and was elected corresponding member of the Bavarian Academy of Sciences. As a guest lecturer he visited the Universities of Oxford, Freiburg, Athens, Siena and Bern.

Troelstra's exceptional ability to examine and extract the essence from the writings of Brouwer, Heyting, Kreisel, Kleene, Gödel and others drove much of his mathematical research. "Formal systems for some branches of intuitionistic analysis" (with Kreisel, 1970) proved the "elimination of choice sequences": each formula without free choice sequence variables is equivalent over CS to a lawlike formula, and CS is an expansion of IDB. In "An Addendum" (1971) Troelstra proved that CS is a notational variant of a conservative extension of Kleene's system I. He provided new models for intuitionistic analysis in "Projections of lawless sequences" I (with van Dalen, 1970) and II (with Gerrit van der Hoeven, 1978), and in "Choice Sequences: a Chapter of Intuitionistic Mathematics" (1970). From a liberal intuitionistic viewpoint he clarified the similarities and differences of the distinct branches of constructivism in his chapter "Aspects of constructive mathematics" for the Handbook of Mathematical Logic (1977).

Many of Troelstra's proofs follow a deceptively simple pattern. Suppose $\mathrm{S} \subsetneq \mathrm{T}$ are intuitionistic formal systems, and $\tau$ is a syntactic translation of formulas $E$ of the language $\mathcal{L}(\mathrm{T})$ into formulas $\tau(E)$ of $\mathcal{L}(\mathrm{S})$ such that both (i) $\vdash_{\mathrm{T}}(E \leftrightarrow \tau(E))$ and (ii) $\vdash_{\mathrm{T}} E \Leftrightarrow \vdash_{\mathrm{S}} \tau(E)$ can be proved finitistically. Then if $\mathcal{L}(\mathrm{T})$ has choice sequence variables but $\mathcal{L}(\mathrm{S})$ does not, the choice sequence variables have been eliminated from sentences of $\mathcal{L}(\mathrm{T})$. If $\tau(E)$ is $E$ for $E$ in $\mathcal{L}(\mathbf{S})$, then $\mathrm{T}$ is a conservative extension of $\mathrm{S}$. If $\tau(0=1)$ is $0=1$, T is consistent relative to $\mathrm{S}$.

Remarkably, Troelstra "axiomatized" Kleene's original realizability interpretations by inventing new principles $\Gamma$ so the pattern is satisfied by $\mathbf{T}=\mathbf{S}+\Gamma$ when $\tau(E)$ expresses " $E$ is realizable." He showed that number-realizability is characterized over Heyting arithmetic by "extended Church's Thesis"

$$
\mathrm{ECT}_{0}: \forall x[A(x) \rightarrow \exists y B(x, y)] \rightarrow \exists e \forall x[A(x) \rightarrow \exists y(\{e\}(x) \simeq y \& B(x, y))],
$$

where $A(x)$ must be "almost negative"(Kleene's term for having no disjunction, and no existential quantifier except immediately in front of a prime formula). Function-realizability is characterized over neutral subsystems of intuitionistic analysis by a corresponding extension of Brouwer's continuous choice principle to almost negative domains $A(\alpha)$, Troelstra's "generalized choice" principle

$$
\mathrm{GC}: \forall \alpha[A(\alpha) \rightarrow \exists \beta B(\alpha, \beta)] \rightarrow \exists \sigma \forall \alpha[A(\alpha) \rightarrow \exists \beta(\{\sigma\}[\alpha] \simeq \beta \& B(\alpha, \beta))],
$$

which we propose to call henceforth "Troelstra's continuous choice principle." These results, which clarified the role realizability plays as a metamathematical principle and have inspired much related research by van Oosten and others, appeared in SLN 344 together with clear expositions of nearly all significant work on intuitionistic formal systems known in Europe or America before 1973.

Especially impressive is the way Anne was able in this book to bring widely distinct methods and areas into view as one unified field of research. He made important observations in passing, e.g., that Kleene's formal treatment of recursive functionals requires only recursive comprehension rather than the "unique choice" principle Kleene assumed, and Heyting's arithmetic of species satisfies a uniformity rule which makes it unsuitable for intuitionistic analysis.

In his metamathematical work not only proof theory, but also various types of model theory, played a role; but his forte was concept analysis. Moerdijk has emphasized Troelstra's synthetic approach to all three, which together led Anne to propose and explore hundreds of 
new axioms and formal systems based on intuitionistic logic and inspired results in category theory, thereby extending the reach of Brouwer's ideas.

In "A note on the fan theorem" (1974) Anne proved that a two-sorted intuitionistic theory with quantifier-free countable choice, Brouwer's fan theorem, and continuous choice is conservative over Heyting arithmetic with respect to arithmetical sentences. He proved a corresponding conservativity result for a classical theory with "weak König's lemma" WKL over Peano arithmetic. Kohlenbach's work on proof mining was partly inspired by these results, and by Anne's theorem that intuitionistic arithmetic of finite types $\mathrm{H}^{\omega}$, and its intensional and extensional variants, are closed under the fan rule: if $\forall f^{1} \exists n^{0} A(f, n)$ is provable, so is $\forall g^{1} \exists m^{0} \forall f \leq g \exists n \leq m A(f, n)$.

Anne wrote the introductory notes to the fundamental articles on the negative translation and the Dialectica interpretation in Volumes I (1986) and II (1990) of Gödel's collected works; and the chapter on realizability for the Handbook of Proof Theory (1998). His textbook "Lectures on Linear Logic" (1992) helped to clarify a subject which was still being developed, and "Basic Proof Theory" (with H. Schwichtenberg, second edition, 2000) is often quoted.

At the University of Amsterdam Anne was sole or principal supervisor for thirteen doctoral students and co-advisor for four others. They include Daniel Leivant, Gerard Renardel de Lavalette, Ieke Moerdijk, Marc Bezem, Ingemarie Bethke, Jaap van Oosten, and Rosalie Iemhoff. His students have contributed significantly to constructive mathematics, category theory, computer science, Martin-Löf type theory, and linear logic. When asked to describe the most rewarding experience of his career, he replied "undoubtedly the contact with my Ph.D. students."

In 1999, on the occasion of his 60th birthday, a commemorative symposium was held in Noordwijkerhout, the Netherlands. It was a gathering of friends; Justus Diller, Martin Hyland, Ulrich Kohlenbach, Helmut Schwichtenberg, Albert Visser, and many others contributed to the associated volume of APAL.

Surprisingly soon afterward Anne announced his retirement and on September 18, 2000, he gave his farewell address. With hindsight this lecture, whose title in English is "Tales of Truth and Sentiment," clearly showed his intentions; his immense intellectual capacities were henceforth directed primarily to natural history. He had always had a deep interest in the subject and a wide knowledge of plants in the Netherlands and abroad, enjoying wildflower expeditions with Olga and Willemien especially. In the time remaining he earned recognition as an authority on natural history travel narratives by writing two books in Dutch on the subject, followed by a comprehensive bibliography in English of books of that genre. With others he also discovered and named three new species of bramble.

When Anne retired his chair was taken over by his lifelong colleague Dick de Jongh, with whom he had coauthored a pioneering paper (written when they were master's students, published in 1966) containing the first definition of a p-morphism as well as the simplest form of duality between Heyting algebra and relational frames. But all through his retirement he remained the authority to whom one could and would go with metamathematical questions, sure of a thoughtful and constructive response generally enlivened by a touch of droll humor.

Anne Troelstra passed away suddenly at the age of 79 on March 7, 2019 in Blaricum, of a brain hemorrhage following a short illness. Life is different for many, now that he is gone.

Dick DE Jongh AND JoAn RAND MoschovaKis 